\title{
Modellbasierte urbane Überflutungsvorsorge
}

\author{
Stefan Reinstaller · Dirk Muschalla
}

Angenommen: 8. Februar 2021 / Online publiziert: 1. März 2021

(C) Der/die Autor(en) 2021

Zusammenfassung Jährlich verursachen zahlreiche konvektive Starkniederschlagsereignisse enorme Schäden und Kosten in den österreichischen Kommunen. Aufgrund ihrer komplexen räumlichen Strukturen und des von der Urbanisierung hervorgerufenen steigenden Versiegelungsgrads ist das Risiko einer Überflutung in urbanen Einzugsgebieten besonders hoch. Daher bedarf es Methoden und Werkzeuge, um mit dem steigenden Überflutungsrisiko in urbanen Einzugsgebieten umgehen zu können. Die Ausweisung von Überflutungsflächen innerhalb eines Einzugsgebiets gilt dabei als wesentliche Grundlage für die Ableitung geeigneter Maßnahmen zur Reduktion des Überflutungsrisikos. Integrierte 1D-2D-Modelle, welche die hydrologische Abflussbildung einerseits sowie den Oberflächenabfluss und den Kanalabfluss hydrodynamisch andererseits berücksichtigen, sind ein zuverlässiges Werkzeug, um die benötigten Zielgrößen - Wasserstand und Fließgeschwindigkeit - zur Ermittlung dieser Überflutungsflächen bestimmen und im Anschluss daran eine Risikoanalyse durchführen zu können. Das Ergebnis sind Überflutungsrisikokarten, die eine wertvolle Planungsgrundlage für die Kommunen darstellen. Die in diesem Beitrag vorgestellte Methodik beschreibt ein Schritt-für-Schritt-Vorgehen, wie Risikokarten in urbanen Einzugsgebieten von Planungsbüros und Kommunen erstellt werden können. Die Methodik ist gegliedert in eine Voruntersuchung zur Identifizierung der neuralgisch wichtigen Stellen, eine Detailanalyse für den Aufbau des integrierten 1D-2D-Modells sowie eine Risikoanalyse zur Erstellung der Überflutungsrisikokarten.

DI S. Reinstaller, BSc ( $\square)$.

Univ.-Prof. Dr.-Ing. D. Muschalla

Institut für Siedlungswasserwirtschaft und Landschaftswasserbau,

Technische Universität Graz, Streymayrgassse 11/I, 8010 Graz, Österreich

stefan.reinstaller@tugraz.at
Schlüsselwörter Urbane

Überflutungen · Integrierte 1D-2D

Modellierung · Risikoanalyse

\section{Model-based urban flood prevention}

Abstract Each year, numerous convective heavy rainfall events cause enormous damage and costs in Austrian municipalities. Due to their complex spatial structures and the increasing degree of imperviousness caused by urbanisation, the risk of flooding in urban catchments is particularly high. Therefore, methods and tools are needed to manage the increasing risk of flooding in urban catchments. The identification of floodplains within a catchment is seen as an essential basis for identifying appropriate measures to reduce the risk of flooding. Integrated 1D2D models, which take into account hydrological runoff generation on the one hand and surface runoff and sewer discharge on the other hand, are a reliable tool for determining the required target variables-water level and flow velocity-for identifying these flood areas and subsequently analysing the risk. The result is flood risk maps that provide a useful planning tool for municipalities. The methodology presented in this paper describes a stepby-step procedure on how risk maps in urban catchments can be produced by planners and municipalities. The methodology is divided into a preliminary analysis to identify the most critical areas, a detailed analysis to build the integrated 1D-2D model, and a risk analysis to produce the flood risk maps.

Keywords Urban flooding - Integrated 1D-2D modelling · Risk analysis

\section{Einleitung}

Als Folge des Klimawandels ist regional ein Anstieg an extremen Starkniederschlagsereignissen $\mathrm{zu}$ verzeichnen, wodurch das Risiko von Überflutungen mit einhergehenden Schäden insbesondere in urbanen Gebieten erhöht wird (O'Donnell und Thorne 2020; Kim et al. 2017; Hammond et al. 2015; Field und Barros 2014). Ein Anstieg der Niederschlagsintensitäten und der Auftrittshäufigkeit kann teilweise in globalen wie auch regionalen Niederschlagsdaten identifiziert werden. In Süddeutschland konnte anhand von Klimaprojektionen eine Zunahme von konvektiven Starkniederschlägen in den Sommerhalbjahren abgeleitet werden (KLIWA 2019). Ähnliche Veränderungen konnten in Österreich durch den Spartacus-Datensatz der Zentralanstalt für Meteorologie und Geodynamik (ZAMG) seit dem Jahr 1961 beobachtet werden. Dieser rechnet mit einer Zunahme an intensiven Starkniederschlagsereignissen bei gleichzeitiger Abnahme von schwächeren oder moderaten Tagesniederschlägen (Chimani et al. 2016). Es ist mit einer 7-\%-Steigerung der Starkniederschläge pro ${ }^{\circ} \mathrm{C}$ Erwärmung zu rechnen, welche bei konvektiven Starkniederschlägen besonders im Süden von Österreich stärker ausfallen kann (ZAMG 2020).

Zusätzlich wird in urbanen Einzugsgebieten das Überflutungsrisiko durch Faktoren wie der Urbanisierung und der daraus folgenden Erhöhung des Versiegelungsgrads verstärkt (Berndtsson et al. 2019). Die Vereinten Nationen (UN) sagen einen globalen Zuwachs der Bevölkerung in urbanen Gebieten weltweit von 3,6 Mrd. (2011) auf 6,7 Mrd. (2050) voraus (68\% der Gesamtbevölkerung) (UN 2018). Ein solcher Trend ist auch in den österreichischen Großstädten zu beobachten. In den Städten Graz und Wien liegt die Wachstumsrate der Bevölkerung zwischen 2010 und 2020 bei $11 \%$ (Statistik Austria 2020), wodurch die Überflutungsproblematik insbesondere in urbanen Einzugsgebieten auch in Österreich erhöht wird.

Obwohl die Gefährdung durch urbane Überflutungen in der öffentlichen Wahrnehmung mittlerweile ausreichend bekannt ist, ist noch kein allgemein anerkanntes Planungsinstrument für den Umgang mit dieser Gefährdung in Österreich vorhanden. Ein solches Instrument wurde in Deutschland im 


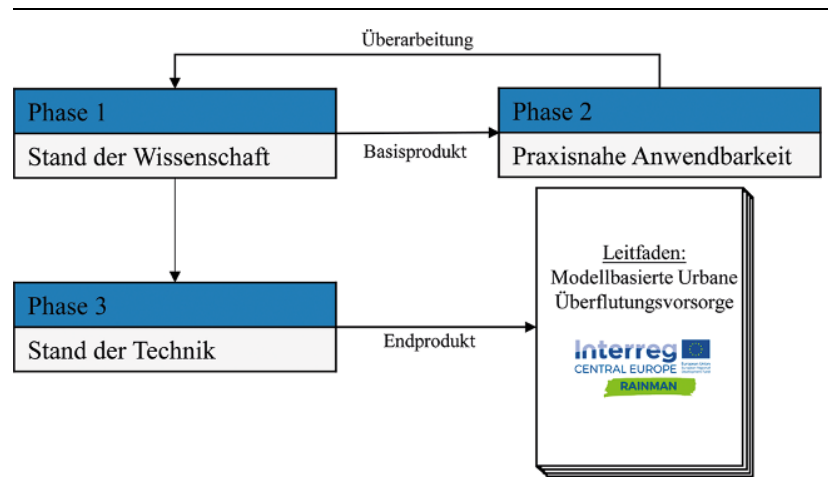

Abb. 1 Methodik zur Erstellung des Leitfadens: Modellbasierte Urbane Überflutungsvorsorge

Jahr 2016 mit dem DWA-Merkblatt 119 „Risikomanagement in der kommunalen Überflutungsvorsorge für Entwässerungssysteme bei Starkregen" vorgelegt (DWA 2016). Um diese Lücke zu schließen, wurde im Zuge des EU-InterregProjekts RAINMAN ein Leitfaden zur modellbasierten Vorsorge von urbanen Überflutungen erarbeitet, welcher in diesem Beitrag vorgestellt wird.

\section{Methodik}

Der in diesem Beitrag vorgestellte Leitfaden ist vorrangig an Planungsbüros und Kommunen gerichtet. Daher wa- ren neben Vertretern der Wissenschaft auch Vertreter kommunaler Einrichtungen (Stadt- und Landesverwaltung) und Planungsbüros bei der Erarbeitung eingebunden. Es resultierte ein 3-Phasen-Konzept für die Erstellung des Leitfadens (Abb. 1).

In der ersten Phase wurde ein Grundlagendokument erarbeitet, welches den Stand der Wissenschaft der urbanen Überflutungsvorsorge repräsentiert. In einer zweiten Phase wurde dieses Dokument anhand zweier Fallstudien angewandt und auf die praxisnahe Anwendbarkeit überprüft. Dabei sollte vor allem die Übertragbarkeit des Leitfadens in die Praxis sichergestellt werden und zu einem optimalen Wissenstransfer aller beteiligten Parteien führen. In einem abschließenden Schritt wurden die gewonnen Erkenntnisse aus den Fallstudien in das finale Dokument eingearbeitet.

Eine Risikoanalyse wird für das Management und die Bewertung von Hochwässern in den Mitgliedstaaten der Europäischen Union gefordert und angewendet (Europäisches Parlament 2007). Diese Methodik ist übertragbar, um urbane Überflutungen bewerten zu können (Scheid 2018; DWA 2016). Risikokarten, welche aus der Kombination der Eintrittswahrscheinlichkeit des urbanen Überflutungsereignisses und der potenziell negativen Folgen auf das betrachtete Schutzgut erstellt werden, sind dabei ein hilfreiches Werkzeug für den vorsorgenden urbanen Überflutungsschutz. Solche Risikokarten sind das Resultat des in dieser Arbeit vorgestellten Leitfadens. Dabei sind folgende Teilschritte notwendig, um Risikokarten zur urbanen Überflutungsvorsorge erstellen zu können (Abb. 2): i) Voruntersuchung (Identifizierung der gefährdeten Stellen (Hot Spots) in einem Einzugsgebiet); ii) Detailanalyse (Aufbau eines urbanen Überflutungsmodells zur Bestimmung der Überflutungsflä-

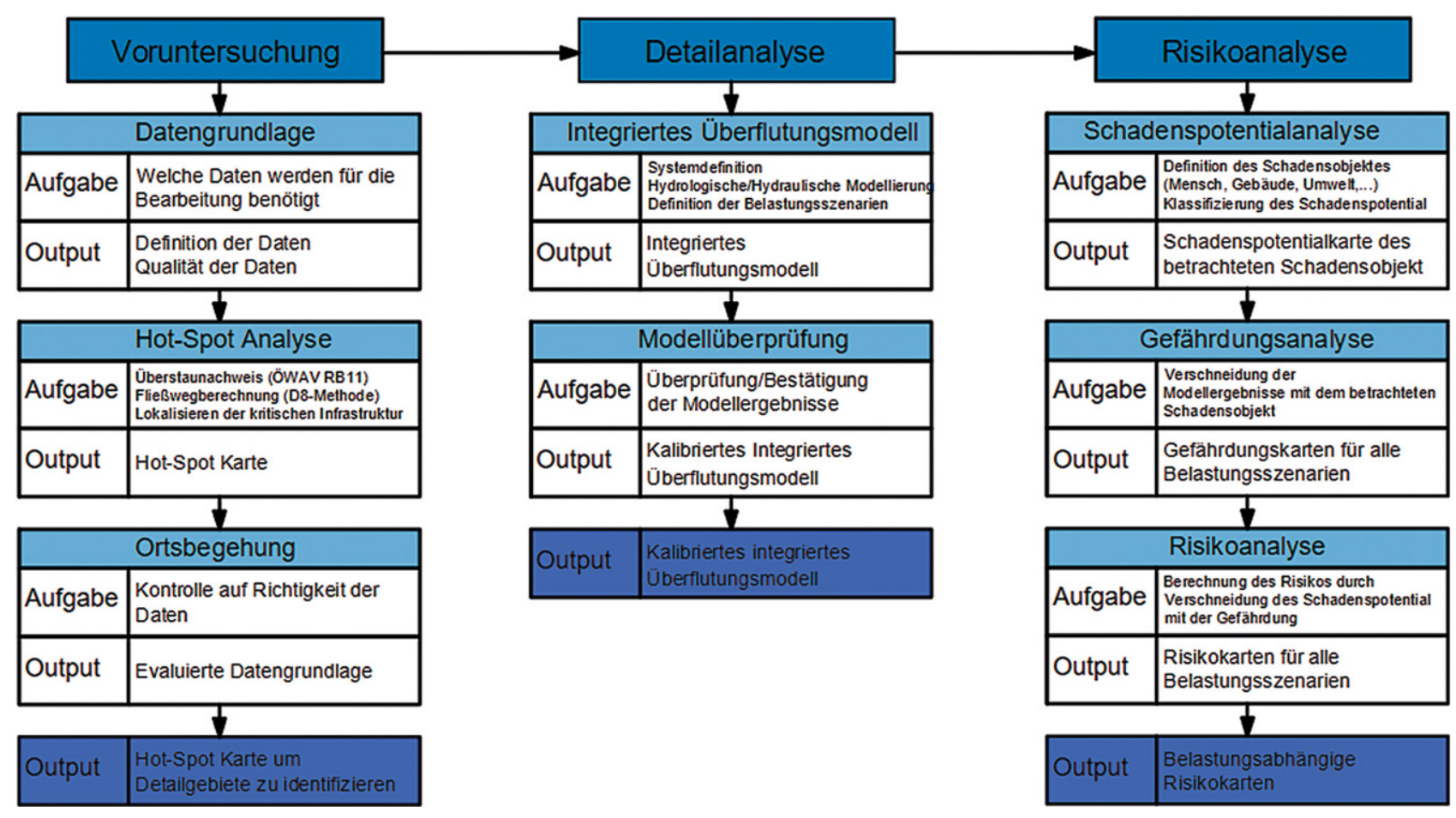

Abb. 2 Ablaufdiagramm zur Erstellung von Risikokarten zur Vorsorge von urbanen Überflutungen (mod. Reinstaller et al. 2020) 
Tab. 1 Datengrundlage für die modellbasierte urbane Überflutungsvorsorge mit der Beschreibung sowie der entsprechenden Relevanz für die Untersuchung

\begin{tabular}{|c|c|c|c|}
\hline Daten & Beschreibung & Anforderung & Relevanz \\
\hline Digitales Höhenmodell & Rasterdatei mit der topografischen Höheninformation in jeder Zelle & $\leq 1 \times 1 \mathrm{~m}$ & Rot: sehr hohe Relevanz \\
\hline Landnutzungsdaten & $\begin{array}{l}\text { Rasterdatei mit dem Landnutzungsindex als Basis für die Zuweisung } \\
\text { hydrologischen Modellparameter }\end{array}$ & $1 \times 1 \mathrm{~m}$ & Orange: hohe Relevanz \\
\hline Niederschlagsdaten & $\begin{array}{l}\text { Niederschlagsradar, Niederschlagsmessung, statische Auswertung von } \\
\text { Niederschlagszeitreihen (ehyd) }\end{array}$ & $\begin{array}{l}\text { Zeitliche Auflösung } \\
<5 \text { min }\end{array}$ & Gelb: mittlere Relevanz \\
\hline Digitaler Kanalkataster & $\begin{array}{l}\text { Vektordatensatz mit allen aktuellen Haltungen, Schächten, und Sonder- } \\
\text { bauwerken für das Kanalmodell }\end{array}$ & Aktueller Stand & Gelb: mittlere Relevanz \\
\hline Digitaler Grundstückskataster & $\begin{array}{l}\text { Vektordatensatz mit den Grundstücksgrenzen sowie Grundstücks- } \\
\text { informationen für die Risikoanalyse }\end{array}$ & Aktueller Stand & Grün: geringe Relevanz \\
\hline $\begin{array}{l}\text { Daten zu vergangenen Über- } \\
\text { flutungen }\end{array}$ & $\begin{array}{l}\text { Schadensdaten oder zeitlich hochaufgelöste Abflussmessungen zur } \\
\text { Modellevaluierung }\end{array}$ & $\begin{array}{l}\text { Mind. } 2 \text { vergangene } \\
\text { Ereignisse }\end{array}$ & Gelb: mittlere Relevanz \\
\hline Orthofoto & Rasterdatei für die Risikoanalyse & Mind. wie DGM & Grün: geringe Relevanz \\
\hline
\end{tabular}

chen); iii) Risikoanalyse (Kombination aus Schadenspotenzialkarten und Gefährdungskarten auf Basis der Überflutungsflächen).

\section{Voruntersuchung}

Das oberste Ziel der urbanen Überflutungsvorsorge ist die Identifikation jener Gebiete innerhalb eines Einzugsgebiets mit einem hohen Überflutungsrisiko. Die Besonderheit eines städtischen Einzugsgebiets liegt vor allem in seiner Heterogenität an verschiedenen möglichen räumlichen Strukturen innerhalb eines Einzugsgebiets. Dabei ist es möglich, dass der Fließvorgang an der Oberfläche von unterschiedlichen Teilprozessen (z. B. urbane Fließgewässer oder Hangwasser) beeinflusst wird, sodass die abflussrelevante Fläche des Einzugsgebietes sehr groß werden kann. Aus diesem Grund wird empfohlen, innerhalb einer Voruntersuchung mit vereinfachten Methoden die neuralgischen Punkte innerhalb eines Einzugsgebiets oder aber auch die problematischen Teileinzugsgebiete innerhalb eines gesamten Stadtgebiets $\mathrm{zu}$ identifizieren. Mit den Ergebnissen können wertvolle Informationen für die weitere Analyse gewonnen werden. So können beispielweise jene Stellen identifiziert werden, an denen eine besonders genaue Datenqualität vorhanden sein muss. Dabei kann es in diesen Bereichen notwendig werden, zusätzliche Daten zu erheben (z.B. Identifikation und Beschreibung von Fließhindernissen wie Mauern, Bordsteinkanten, Garageneinfahrten usw.). Die vorgestellte Methodik lässt sich auch auf ein gesamtes Stadtgebiet übertragen, um die besonders gefährdeten Teileinzugsgebiete innerhalb des gesamten Stadtgebiets zu identifizieren. Dadurch kann ein unnötig hoher Rechenaufwand vermieden und vermeidbare hohe Kosten eingespart werden, da nicht für das gesamte Stadtgebiet ein urbanes Überflutungsmodell erstellt werden muss.

Die Voruntersuchung gliedert sich in drei wesentliche Teile: i) Definition der notwendigen Datengrundlage, ii) HotSpot-Analyse: GIS-basierte Fließweganalyse sowie Überstaunachweis des Kanalsystems, iii) Ortsbegehung zur Validierung der Ergebnisse der HotSpot-Analyse sowie der Datengrundlage. Als Zwischenergebnis werden sogenannte Hot-Spot-Karten erstellt, welche alle Ergebnisse der Voruntersuchungen beinhalten und zusammenfassen.

\subsection{Datengrundlage, Datenerhebung und Datenverfügbarkeit}

Aufgrund der Tatsache, dass eine urbane Überflutung von einer Vielzahl an Teilprozessen beeinflusst wird, ist eine hohe Anzahl an Daten notwendig, um ein urbanes Überflutungsmodell aufbauen, evaluieren und analysieren $\mathrm{zu}$ können (Tab. 1). Die Datentypen zur Modellerstellung werden in Eingabedaten (z.B. Digitales Geländemodell, Landnutzungsdaten), Systembelastungen (z.B. Regendaten), und Modellparameter (z. B. hydraulische und hydrologische Parameter wie Rauigkeit oder Versieglungsgrad) unterschieden. Die benötigte Genauigkeit der Daten hängt davon $a b$, in welchem Maß die Daten das Abflussverhalten beeinflussen können. Eine Vielzahl an Untersuchungen zeigt, dass die Qualität des verwendeten digitalen Geländemodells einen entscheidenden Einfluss auf das Abflussverhalten an der Oberfläche hat (Hawker et al. 2018; Scheid 2018). Zusätzlich wird der Qualität der Landnutzungsdaten eine hohe Wichtigkeit zug- ordnet, da diese einen maßgeblichen Einfluss auf die Wahl der hydrologischen Modellparameter haben.

Neben den Daten zur Modellerstellung müssen die notwendigen Daten zur Evaluierung und Bewertung des Modells definiert werden. Dazu können zum einen vorhandene Abflussmessungen im Kanal oder in einem urbanen Fließgewässer und zum anderen örtlich bezogene Schadensdaten (z.B. Dokumentationen von Einsatzkräften) verwendet werden. Ebenso können auch einfache Analysen von Social-Media-Beiträgen Hinweise auf das Abflussverhalten an der Oberfläche geben (Matzinger et al. 2019).

Alle notwendigen Daten sollten bereits in diesem frühen Stadium der Untersuchung definiert werden, da diese bereits teilweise in der nachfolgenden Hot-Spot-Analyse benötigt werden. $\mathrm{Zu}-$ sätzlich können etwaige Lücken in der Datenverfügbarkeit auch für spätere Analysen (Detail- und Risikoanalyse) sehr früh identifiziert werden, sodass in diesem Fall rechtzeitig nachgebessert werden kann (z.B. im Zuge der Ortsbegehung).

\subsection{Hot-Spot-Analyse, Fließweganalyse und Überstaunachweis}

Die topografische Fließweganalyse basiert in den derzeit häufig implementierten Algorithmen auf dem „deterministic eight neighbors“ Ansatz (D8Methode), welcher jeder Zelle eine von acht möglichen Fließrichtungen zuordnet (Fairfield und Leymarie 1991). Im Anschluss wird anhand einer Abflussakkumulation bestimmt, wie viele Zellen in jeweils eine Zelle entwässern. Dadurch können neben der Größe des hydrologischen, oberirdischen Einzugs- 


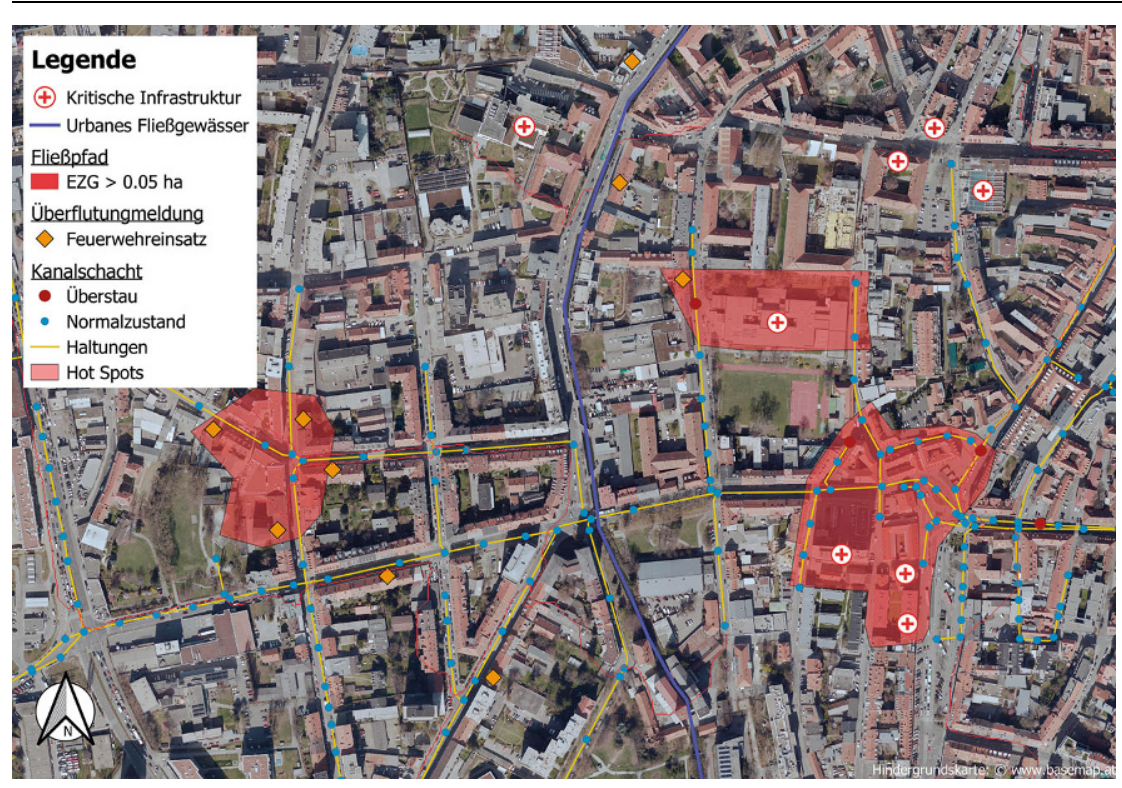

Abb. 3 Beispielhafte Darstellung einer Hot-Spot-Karte als Endprodukt der Voruntersuchung im Zuge der urbanen Überflutungsvorsorge

gebiets auch die Hauptfließpfade bestimmt und klassifiziert werden.

Die Datengrundlage für diese Methode ist ein möglichst hochaufgelöstes Digitales Geländemodell (DGM) des Einzugsgebiets. Dabei ist es notwendig, möglichst alle Hindernisse, die das Abflussverhalten beeinflussen, in das DGM zu integrieren. Besonders Brücken, Durchlässe, Mauern und Gebäude stellen neuralgisch wichtige Punkte in diesem Zusammenhang dar. Ein gutes und aktuelles Orthofoto wird daher neben einem hochaufgelösten DGM ebenfalls benötigt.
Diese sind zumindest in den größeren Gemeinden landesweit frei verfügbar (z. B. basemap.at) und können mit den notwendigen Informationen aus den GIS-Portalen der jeweiligen Bundesländer gekoppelt werden (GIS-Steiermark 2018).

Da urbane Überflutungen neben den hydrologischen Prozessen an der Oberfläche auch von unterirdischen Prozessen beeinflusst werden können, sollte die Voruntersuchung auch die Auswirkung sowie den Einfluss des Kanalsystems auf das Abflussverhalten berücksichtigen. Ein digitaler Kanal- kataster sowie ein hydrodynamisches Kanalnetzmodell ist dabei zwingend erforderlich, um einen Überstaunachweis nach ÖWAV-Regelblatt 11 (2009) durchführen zu können. Ist die Datengrundlage im Untersuchungsgebiet nicht gegeben, muss der Zeitaufwand abgeschätzt werden, um diese Daten $\mathrm{zu}$ erheben beziehungsweise ein hydrodynamisches Kanalnetzmodell aufzubauen. Dies kann mitunter einen beträchtlichen Mehraufwand bedeuten. Die identifizierten Schächte im Einzugsgebiet, an denen mit einem häufigen Überstau gerechnet werden kann, werden anschließend in den Hot-SpotKarten erkenntlich gemacht (Abb. 3).

Eine weitere Information die die Hot-Spot-Karte enthalten sollte, sind Bauwerke der kritischen Infrastruktur sowie vulnerable Gebäude wie Schulen, Kindergärten oder Altenheime. Neben dem Flächenwidmungsplan der jeweiligen Gemeinde bieten offene OnlinePlattformen wie openstreetmap eine gute Datengrundlage, um solche Gebäude identifizieren zu können. Die Gebäudenutzung wird ebenfalls berücksichtigt, da diese Informationen für die spätere Risikoanalyse essenziell sind.

\subsection{Ortsbegehung}

Im Zuge einer umfassenden Ortsbegehung sollen die Ergebnisse der HotSpot-Analyse verifiziert und etwaige fehlende Daten ergänzt werden. Die Erhebung von Bruchkanten, Garageneinfahrten sowie Durchlässen sei in die-
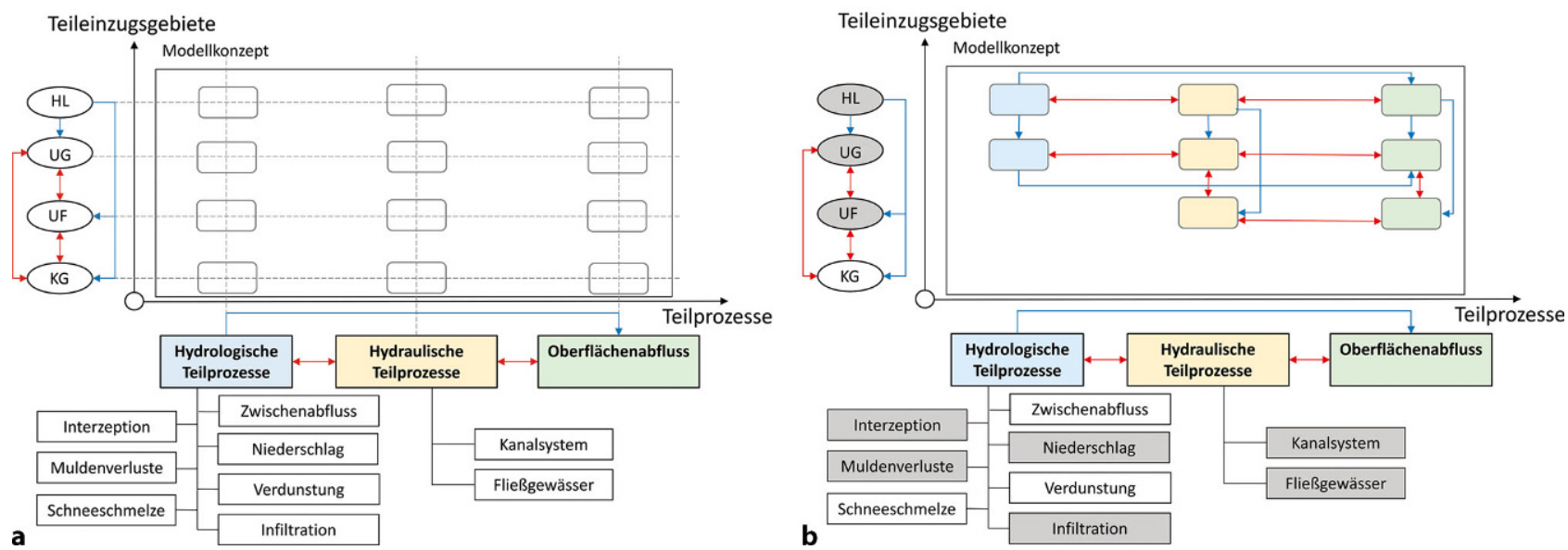

Abb. 4 MethodischerVorgang der zweidimensionalen Modellintegration(a) sowie eine fiktive Umsetzung durchAnnahmenund Vereinfachungen (b) bezüglich abflussrelevanter Teilprozesse und von der räumlichen Struktur des Untersuchungsgebiets (Hanglage (HL), Urbanes Gebiet inkl. Kanalsystem (UG), Urbanes Fließgewässer (UF), Küstengebiet-und Ufergebiete (KG)) abhängigen Vorkommen der Teileinzugsgebiete (grau hinterlegte Teilprozesse und Teileinzugsgebiete stellen die Anforderungen für das zu erstellende urbane Überflutungsmodell dar) 


\begin{tabular}{|c|c|c|c|c|}
\hline Klasse & Beschreibung & $\begin{array}{l}\text { Kombination Grenzwert } \\
(\mathrm{H} * \mathrm{~V})\end{array}$ & $\begin{array}{l}\text { Wasserstand Grenzwert } \\
\text { (H) }\end{array}$ & $\begin{array}{l}\text { Geschwindigkeit Grenzwert } \\
\text { (V) }\end{array}$ \\
\hline G1 & Sicher für Fahrzeug, Mensch und Gebäude & $\leq 0,3$ & $0,3[\mathrm{~m}]$ & $2[\mathrm{~m} / \mathrm{s}]$ \\
\hline G2 & Unsicher für kleine Fahrzeuge & $\leq 0,6$ & $0,6[\mathrm{~m}]$ & $2[\mathrm{~m} / \mathrm{s}]$ \\
\hline G3 & Unsicher für Fahrzeuge, Kinder und ältere Menschen & $\leq 0,6$ & $0,6[\mathrm{~m}]$ & $2[\mathrm{~m} / \mathrm{s}]$ \\
\hline G4 & Unsicher für Fahrzeug und Menschen & $\leq 1,0$ & $1,0[\mathrm{~m}]$ & $2[\mathrm{~m} / \mathrm{s}]$ \\
\hline G5 & $\begin{array}{l}\text { Unsicher für Fahrzeuge, Menschen und Gebäude in } \\
\text { Leichtbauweise }\end{array}$ & $\leq 4,0$ & $4,0[\mathrm{~m}]$ & $4[\mathrm{~m} / \mathrm{s}]$ \\
\hline G6 & Unsicher für alle Fahrzeuge, Menschen und Gebäude & - & - & - \\
\hline
\end{tabular}

Tab. 3 Zweidimensionale Kontingenztabelle für die qualitative Modellevaluierung

\begin{tabular}{llll}
$\begin{array}{l}\text { Beschreibung des } \\
\text { Überflutungsereignisses } \\
\text { (Wiederkehrperiode, } \\
\text { Dauer) }\end{array}$ & & Reale Beobachtung (Schadensmeldungen) \\
& & Positiv & Negativ \\
\hline Simulation & Positiv & $\begin{array}{l}\text { Richtig positiv } \\
\text { (RP) }\end{array}$ & $\begin{array}{l}\text { Falsch negativ } \\
\text { (FN) }\end{array}$ \\
& Negativ & $\begin{array}{l}\text { Falsch positiv } \\
\text { (FP) }\end{array}$ & $\begin{array}{l}\text { Richtig negativ } \\
\text { (RN) }\end{array}$ \\
& & &
\end{tabular}

sem Kontext beispielhaft angeführt. Die Erkenntnisse aus der Begehung werden im Anschluss in die bestehenden HotSpot-Karten zusätzlich eingearbeitet.

\section{Detailanalyse}

Im Zuge der Detailanalyse wird ein integriertes urbanes Überflutungsmodell aufgebaut, welches in der Lage ist, die ereignisbezogenen Überflutungsflächen zu berechnen. Da sich der Fokus dieses Beitrags auf urbane Überflutungen konzentriert, muss neben einem klassischen Niederschlags-Abflussmodell für die Oberfläche auch ein Kanalnetzmodell erstellt und gekoppelt werden, um die Überflutungsflächen bestimmen zu können. Dieser Ansatz wird in der Literatur mit dem Begriff 1D-2D urbanes Überflutungsmodell beschrieben (Schmitt et al. 2004).

Aufgrund der enormen Vielzahl an kommerziellen, Open-Source- und Freeware-Produkten auf dem Markt ist die richtige Modellauswahl kein einfacher Prozess. Zudem weist jedes Modell Unsicherheiten auf, welche durch unterschiedlichste Quellen entstehen. Guzman et al. (2015) teilen die Unsicherheitsquellen in folgende Klassen ein: i) Unsicherheiten in der Modellstruktur; ii) Unsicherheiten in den Eingangsdaten (Landnutzung, DGM, Regendaten); iii) Unsicherheiten der Evaluierungsdaten (z.B. Messfehler bei Abflussmessungen). All diese Unsicherheiten finden sich in unterschiedlichsten Phasen der Detailanalyse wieder. So muss die Unsicherheit der Modell- struktur in der Modellerstellung und Auswahl berücksichtigt werden. Genauso müssen alle verwendeten Daten und Modellparameter stets kritisch betrachEinfluss auf die gesuchte Zielgrößen (im Fall der Überflutungsmodellierung: Wasserstand und Fließgeschwindigkeit) haben können. Daher sollten die Unsicherheiten in jedem Teilschritt der Detailanalyse berücksichtigt werden. Beispielweise können die Unsicherheiten der Modellparameter durch eine vereinfachte Sensitivitätsanalyse analysiert werden, um die unrealistische Definition eines Parameters (z. B. Rauigkeiten) zu vermeiden.

\subsection{Modellentwicklung und Modellstruktur}

Die Modellintegration ist nach Rauch et al. (2002) die Kopplung zweier physikalischer Prozesse innerhalb eines Systems. Diese Definition kann im Rahmen der urbanen Überflutungsvorsorge übernommen werden, da eine Vielzahl an physikalisch basierten Teilprozessen das Abflussverhalten an der Oberfläche beeinflussen und daher in einem Modell integriert werden müssen (z.B. bidirektionaler Austausch zwischen überstautem Kanalsystem und dem Oberflächenabfluss).

Da die Anzahl der abflussrelevanten Teilprozesse sehr hoch sein kann, werden diese zur besseren Übersicht in Prozessgruppen zusammengefasst. Die erste Gruppe fasst alle hydrologischen Teilprozesse zusammen, um die tet werden, da diese einen erheblichen
Abflussbildung bestimmen zu können. Das daraus entstehende Abflussvolumen kommt anschließend entweder im Fließgewässer, im Kanalsystem oder an der Oberfläche selbst zum Abfluss. Daher wird als zweite Gruppe eine hydraulische Prozessgruppe definiert, welche das Fließverhalten an der Oberfläche, im Fließgewässer sowie im Kanalsystem bestimmt.

Die folgenden Teilprozesse können überflutungsrelevant sein und müssen daher in einem urbanen Überflutungsmodell eventuell berücksichtigt werden: i) Oberflächenabfluss; ii) Kanalabfluss; iii) Fließgewässerabfluss; iv) Infiltration; v) Verdunstung, vi) Muldenspeicher; vii) Interzeption; viii) Schneeschmelze; ix) Niederschlag; x) Zwischenabfluss. Der Oberflächenabfluss wird als der maßgebliche Teilprozess identifiziert und kann daher in der urbanen Überflutungsmodellierung nicht vernachlässigt werden.

Die Wirkung jedes Teilprozesses auf das Abflussverhalten an der Oberfläche ist je nach struktureller Eigenschaft des Einzugsgebiets in seiner Ausprägung unterschiedlich. Ein urbanes Einzugsgebiet kann eine große strukturelle Heterogenität aufweisen. Es ist daher sinnvoll, ein urbanes Einzugsgebiet in einem ersten Schritt in Teileinzugsgebiete $\mathrm{zu}$ unterteilen (z.B. i) Hanglage (HL), ii) Urbanes Gebiet inkl. Kanalsystem (UG), iii) Urbanes Fließgewässer (UF), iv) Küsten- und Ufergebiete (KG)). Anschließend werden die abflussrelevanten Interaktionen zwischen den Teileinzugsgebieten definiert, welche wie bei den Teilprozessen bidirektional wie auch unidirektional sein können. Abschließend muss der Einfluss der räumlich strukturellen Eigenschaften auf jeden einzelnen physikalischen Teilprozess bestimmt werden. Dabei können in Abhängigkeit von der Fragestellung und der räumlich strukturellen Eigenschaften einer oder mehrere Teilprozesse vernachlässigt werden. 


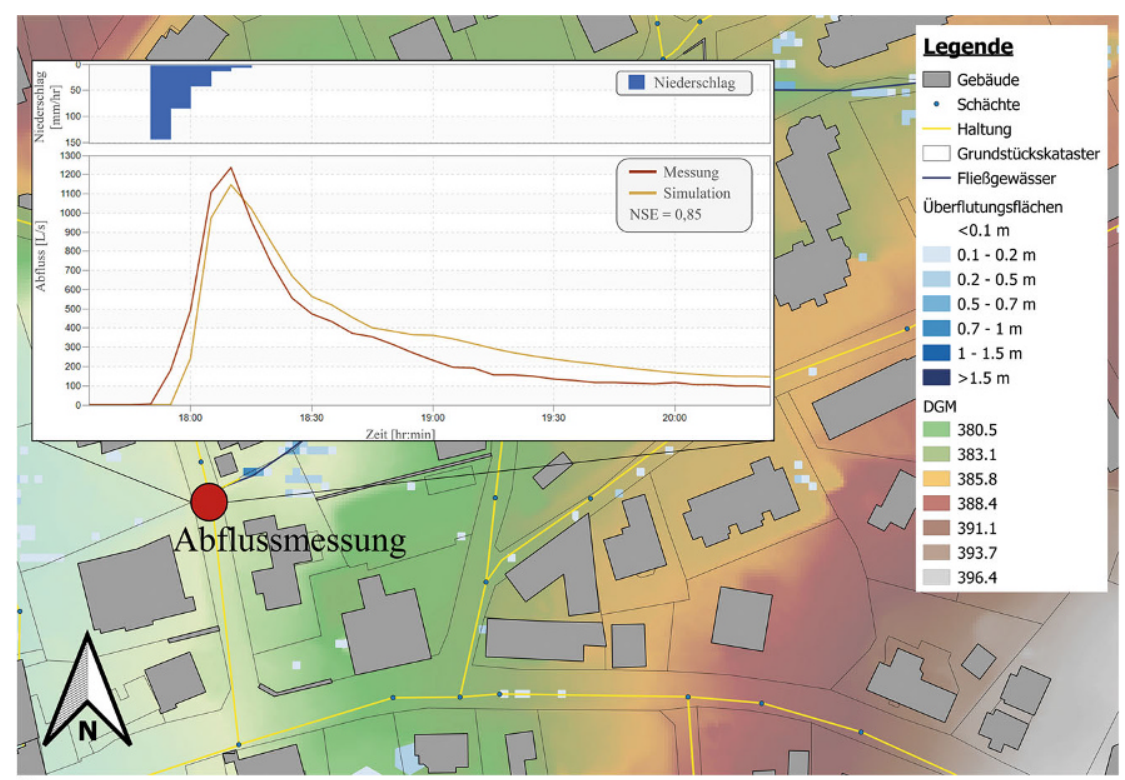

a

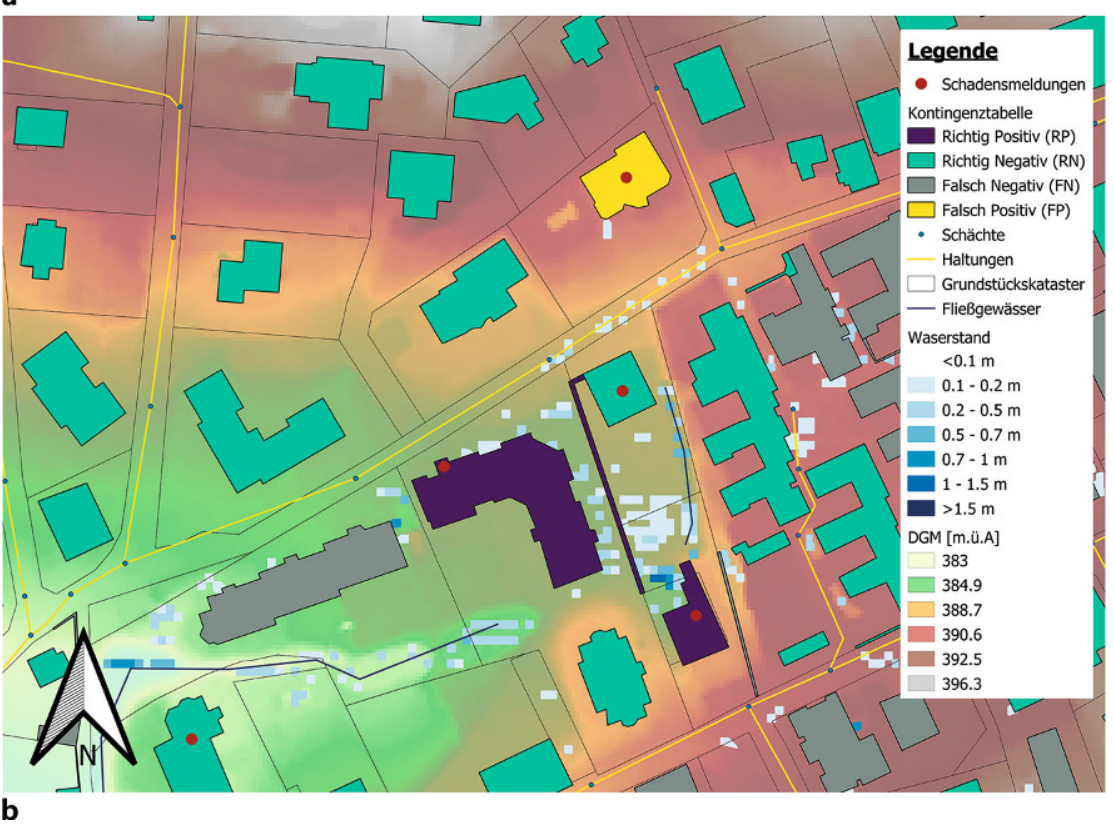

Abb. 5 Beispielhafte Darstellung einer quantitativen (a) und qualitativen Modellevaluierung (b)

Beispielweise können die Teilprozesse Verdunstung und Zwischenabfluss bei konvektiven Starkregenereignissen vernachlässig werden, da diese eine lange Zeitspanne benötigen, um einen Einfluss auf das Abflussverhalten an der Oberfläche oder im Fließgewässer im Starkregenfall zu haben (Mosley und McKerchar 1993). Im Gegensatz dazu können diese Teilprozesse bei der Modellierung von Überflutungen aufgrund starker Niederschläge über einen langen Zeitraum, welche bei fluvialen Überflutungen maßgeblich sind, nicht vernachlässigt werden. Allerdings kön- abflussvolumen und höheren Fließgeschwindigkeiten auftritt.

Es ist daher schwierig, ein spezifisches Modell für den komplexen Vorgang der urbanen Überflutung zu empfehlen, da die Auswahl stark vom Charakter des Niederschlagsereignisses (lange intensive Starkniederschläge über das ganze Jahr oder kurze konvektive Starkniederschläge in den Sommermonaten) sowie von der Struktur des jeweiligen Einzugsgebiets abhängig ist. Aus diesem Grund wird an dieser Stelle das Erstellen eines geeigneten Modellierungskonzepts beschrieben, nicht aber die theoretischen Grundlagen sowie die Vor-und Nachteile eines oder mehrere Modelle diskutiert.

Zusammengefasst ist die hier vorgestellte Methodik von der herkömmlichen Modellintegration zu unterscheiden, da diese neben Integration zwischen den Teilprozessen auch eine Integration zwischen den im urbanen Einzugsgebiet vorkommenden Teileinzugsgebieten beinhaltet. In diesem Zusammenhang wird der Begriff der zweidimensionalen Modellintegration eingeführt. Dabei beinhaltet die erste Dimension die Integration der physikalisch basierten und abflussrelevanten Teilprozesse, die zweite Dimension umfasst die Integration der Teilprozesse zwischen den im Einzugsgebiet auftretenden Teileinzugsgebieten (Abb. 4).

Sind das Modellierungskonzept sowie die semantische Beziehung der einzelnen Teilprozesse in den vorkommenden Teileinzugsgebieten definiert, muss für jeden Prozess ein geeigneter Modellansatz gewählt werden, welcher in der Lage ist, diesen realitätsnah abzubilden. Insbesondere der geeignete Ansatz für das Fließverhalten an der Oberfläche wurde in vergangenen Studien bereits zahlreich diskutiert, beispielweise in der von Hunter et al. (2008) vorgestellten Modell-Benchmark-Studie. Dabei hat die Qualität des digitalen Geländemodells (DGM) eine hohe Bedeutung, da auf diesem die räumliche Diskretisierung (Netzerstellung) basiert (Hawker et al. 2018; Wang et al. 2018). Aus diesem Grund ist ein genaues DGM für die urbane Überflutungsmodellierung essenziell. Mit einer möglichst hohen Qualität des DGMs werden gleichzeitig die Unsicherheiten in den normalerweise betrachteten Zielgrößen (Wasserstand und Fließgeschwindigkeit) reduziert. Die Anwendung der oben vorgestellten Methodik zur Erstellung eines Modellkonzepts re- 
Tab. 4 Mögliche Klassifizierung des Schadenspotenzials und der Überflutungsgefahr (mod. DWA2016)

\begin{tabular}{|l|l|l|l} 
Klasse & $\begin{array}{l}\text { Beurteilung Schadenspo- } \\
\text { tenzial }\end{array}$ & Beschreibung der Gebäudenutzung & $\begin{array}{l}\text { Beurteilung Überflutungs- } \\
\text { gefahr }\end{array}$ \\
\hline 1 & Gering & Kleingartenbebauung, Parks, und Grünflächen & $<0,1[\mathrm{~m}]$ \\
\hline 2 & Mäßig & Wohnbau ohne Untergeschoss, Einzelhandel und Kleingewerbe & $0,1-0,3[\mathrm{~m}]$ \\
\hline 3 & Hoch & Schule, Hochschule (Universitäten), bewohnter Wohnbau mit Untergeschoss & $0,3-0,5[\mathrm{~m}]$ \\
\hline 4 & Sehr hoch & $\begin{array}{l}\text { Kindergarten, Unterführungen, Krankenhaus, Rettungsdienst, Altenheim, U-Bahn-Statio- } \\
\text { nen, Gebäude der Kritischen Infrastruktur, Tiefgaragen }\end{array}$ & $>0,5[\mathrm{~m}]$ \\
\hline
\end{tabular}

duziert die Modellunsicherheiten in der Modellstruktur durch den Fokus auf die abflussrelevanten Teilprozesse.

\subsection{Simulationsszenarien}

Ein wichtiger Teil der Detailanalyse ist die Auswahl geeigneter Simulationsszenarien, um ein robustes urbanes Überflutungsmodell aufzubauen. Damit die Aussagekraft des Modells garantiert werden kann, sind mehrere Simulationsszenarien mit unterschiedlichen Dauerstufen und Wiederkehrwahrscheinlichkeiten nötig. Im österreichischen Wasserrecht wird für die Gefahrenzonen-Ausweisung von Hochwässern eine Wiederkehrwahrscheinlichkeit von 30 Jahren (hohe Wahrscheinlichkeit), 100 Jahren (mittlerer Wahrscheinlichkeit) und 300 Jahren (geringe Wahrscheinlichkeit) gefordert (BMLFUW 2016). Eine solche Einteilung kann auch für urbane Überflutungen übernommen werden, allerdings kann davon ausgegangen werden, dass die Überflutungsflächen zwischen einer Wiederkehrperiode von 100 und 300 Jahren nur mehr geringe Änderungen erfahren. Außerdem ist in Abhängigkeit von der Lage des Einzugsgebiets (Innenstadt oder Randlage) eine Wiederkehrperiode von 10 Jahren oder kleiner für die Dimensionierung der Kanalisation in Österreich gefordert (ÖWAV 2009). Das DWA-Merkblatt 119 (DWA 2016) empfiehlt neben einer Wiederkehrperiode von 30 und 100 Jahren noch eine weitere mit 50 Jahren. Aus diesem Grund werden Wiederkehrperioden von $10,30,50$, und 100 Jahren für die Betrachtung von urbanen Überflutungen empfohlen.

Neben der Wiederkehrperiode ist auch die geforderte Niederschlagsverteilung zu definieren. Dabei wird zwischen Bemessungsereignissen, welche auf statistischen Auswertungen beruhen, und realen gemessenen Ereignissen unterschieden. Die ehyd-Plattform (BMLFUW 2011) bietet dabei eine offene statistische Datengrundlage an, um Bemessungsniederschläge wie den $\mathrm{Eu}$ -
ler-Modellregen Typ II oder die mittenbetonte Niederschlagsverteilung erstellen zu können. Der Vorteil von Bemessungsniederschlägen liegt zum einen in der landesweiten Verfügbarkeit der benötigten Niederschlagsdatenauswertung und zum anderen an den kurzen, benötigten Simulationszeiten aufgrund der kurzen Dauerstufen, welche bei der Betrachtung von urbanen Überflutungen zu erwarten sind. Allerdings kommt es bei der Anwendung von Modellregen oftmals zu Überschätzungen der Überflutungsflächen, da diese Niederschlagsverteilung nicht für den Oberflächenabfluss, sondern für den Abfluss im Kanal oder Fließgewässer erstellten worden sind. Daher ist es anzustreben, neben Bemessungsniederschlägen auch Szenarien basierend auf realen Niederschlagsdaten mit realen Verteilungen zu erstellen und miteinander zu vergleichen. Dies ist zwar im Moment nur in vereinzelten Fällen möglich, wird jedoch künftig durch den Ausbau von Messnetzen wie dem WegenerNet des Wegener-Centers für Klima und Globalen Wandel der Universität Graz (Kirchengast et al. 2014) sowie Niederschlagsradarsystemen häufiger möglich werden. Ein weiterer Grund für die Anwendung realer Niederschlagsverteilungen ist die Forderung nach einer bestmöglichen Modellevaluierung. Diese benötigt neben Schadensdaten oder Abflussmessungen auch die reale Niederschlagsverteilung, um ein urbanes Überflutungsereignis realitätsnahe $\mathrm{zu}$ simulieren. Um die Robustheit des Modells garantieren $\mathrm{zu}$ können, werden mindestens zwei solcher realer oder historischer Überflutungsereignisse mit einer Wiederkehrperiode von größer als 20 Jahren benötigt.

\subsection{Modellevaluierung}

Der abschließende Schritt jedes Modellierungsprozesses ist die Modellevaluierung. Dabei werden die Simulationsergebnisse mit dem realen Systemverhalten verglichen. Die Kalibrierung mit einer anschließenden Validierung ba- sierend auf statischen Zielfunktionen ist die anerkannte Methode, um ein Modell bestmöglich zu evaluieren. Im Kontext der Überflutungsmodellierung sind der Wasserstand und die Fließgeschwindigkeit auf der Oberfläche die gesuchten Zustandsvariablen, welche betrachtet werden müssen. Ebenfalls kann der Abfluss im Kanalsystem sowie in einem Fließgewässer im Modellgebiet für die Evaluierung verwendet werden. Dies bietet die Möglichkeit, Abflussmessungen an neuralgischen Stellen im Kanalsystem oder im Fließgewässer zu betrachten, um in weiterer Folge die Simulationsergebnisse mit den gewonnenen Abflussdaten quantitativ vergleichen zu können. Ein großer Vorteil einer Abflussmessung ist die hohe zeitliche Auflösung. Dadurch kann die zeitliche Verteilung des Überflutungsereignisses sehr gut berücksichtigt werden. Die Nachteile einer Abflussmessung sind die hohen Anschaffungs-und Betriebskosten der Messtechnik sowie der zeitliche Aufwand für die Erstellung eines geeigneten Datensatzes zur Kalibrierung und Validierung des Modells. Es kann nicht garantiert werden, dass im Messzeitraum ein Überflutungsereignis stattfinden wird, welches für die Modellevaluierung verwendet werden kann, da die geforderten Wiederkehrperioden sehr hoch sind ( $>20 \mathrm{a})$. Mit einer quantitativen Modellevaluierung wird darüber hinaus nur eine Stelle im gesamten Einzugsgebiet betrachtet. Mit nur einer Abflussmessung wird die räumliche Variabilität des Systemverhaltens nicht berücksichtigt, dies kann zu Fehlaussagen bezüglich der Modellgenauigkeit führen. Die naheliegende Lösung dieses Problems wären Messungen des Wasserstands auf der Oberfläche an mehreren maßgeblichen Stellen im Einzugsgebiet. Dabei stößt die Messtechnik derzeit noch an ihre Grenzen, da es schwierig ist, Wasserstand, Abfluss oder Fließgeschwindigkeit ohne definierten Fließquerschnitt zu bestimmen. Außerdem müssten die Messungen auf der freien Oberfläche implementiert werden. Eine quantitati- 

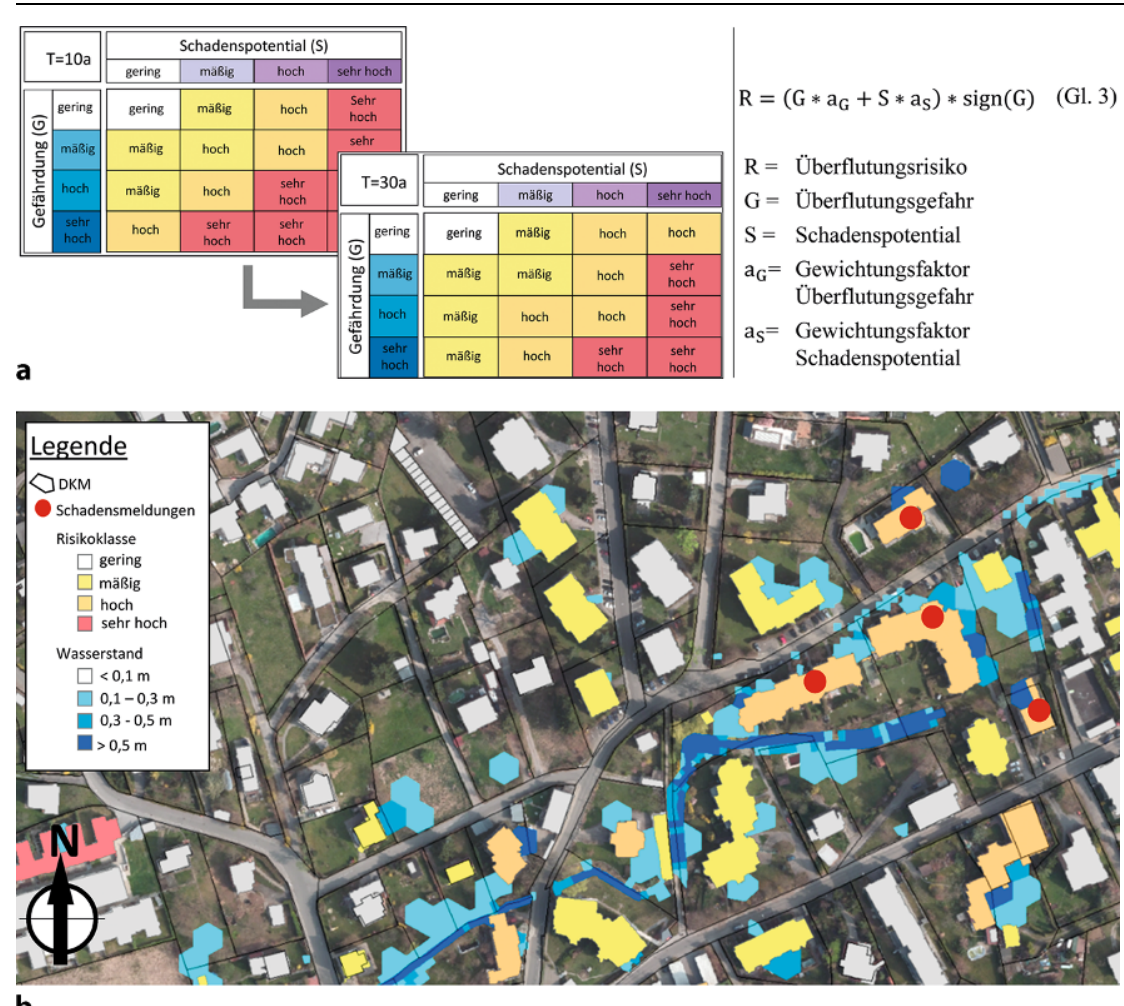

b

Abb. 6 Berechnung des Überflutungsrisikos nach Scheid (2018) zur Bestimmung der Risikomatrix einer zugewiesener Wiederkehrperiode für die Erstellung einer Überflutungsrisikokarte (mod. Reinstaller et al. 2020) als Endprodukt der urbanen Überflutungsvorsorge

ve Modellevaluierung ist daher nur in wenigen Fällen geeignet, um ein urbanes Überflutungsmodell beurteilen zu können. Es müssen andere Methoden zur Modellevaluierung herangezogen werden.

Eine geeignete Methodik, um die Modellgüte $\mathrm{zu}$ analysieren, ist die auf Schadensdaten basierende qualitative Evaluierung (Bennett et al. 2013). Mit dieser Methodik werden die aus den Simulationen resultierenden Überflutungsflächen mit realen Schadensdaten verglichen. Auf Grundlage der Modellergebnisse werden die Schadensobjekte (Gebäude) binär bewertet $(0=$ kein Schaden, $1=$ Schaden $)$. Dafür wird eine Grenzbedingung für die Modellvariablen (Wasserstand und Fließgeschwindigkeit) benötigt. Hier kann auf Schadensfunktionen zurückgegriffen werden, die einen funktionalen Zusammenhang zwischen Modellvariablen und Schaden herstellen. Smith et al. (2014) teilen die Überflutungsgefährdung auf Gebäude, Fahrzeuge und Menschen in sechs Klassen ein, wobei eine Kombination von Wasserstand und Fließgeschwindigkeit als Grenzbe-
Verfügung stehenden qualitativen Zielgrößen.

$$
\begin{aligned}
& \mathrm{A}=\frac{\mathrm{RP}+\mathrm{RN}}{(\mathrm{RP}+\mathrm{RN}+\mathrm{FP}+\mathrm{FN})} \\
& \mathrm{HR}=\frac{\mathrm{RP}}{(\mathrm{RP}+\mathrm{FN})}
\end{aligned}
$$

Eine Schwierigkeit der qualitativen Modellevaluierung ist die Generierung eines Datensatzes der realen Überflutungsschäden. Hierfür kann beispielweise auf Einsatzmeldungen von Blaulicht-Organisationen, aber auch auf Meldungen in Social Media (Facebook, Twitter, Instagram) sowie auf anonymisierte Versicherungsdaten zurückgegriffen werden. Außerdem kann der zeitliche Verlauf des Überflutungsereignisses nur indirekt bewertet werden. Ein Vorteil dieser Methodik ist, dass die räumliche Variabilität des Systemverhaltens mitberücksichtigt wird und daher die Aussagen bezüglich der Modellgenauigkeit realitätsnahe sind. Zusätzlich ist die Unsicherheit der Schadensdaten nicht so hoch wie bei einer Abflussmessung, da nur ein Schaden oder kein Schaden an einem Objekt bewertet wird. Die Größe des Schadens kann durch eine Erhöhung der Grenzbedingung bezogen auf die Gefährdungsklassen berücksichtigt werden.

Zusammengefasst sind sowohl die quantitative als auch die qualitative Modellevaluierung in der Lage, ein urbanes Überflutungsmodell zu bewerten (Abb. 5). Beide vorgestellten Methoden weisen Vor- und Nachteile auf. Daher ist es schwierig, eine der beiden Methoden für die Evaluierung von urbanen Überflutungsmodellen generell zu empfehlen. Die quantitative Modellevaluierung ist in der Lage, den zeitlichen Verlauf des Überflutungsereignisses zu berücksichtigen und eignet sich, um das Modell zu kalibrieren und zu validieren. Allerdings kann die räumliche Variabilität der Überflutungsflächen nicht bewertet werden. Dies ist klar der Vorteil der qualitativen Modellevaluierung. Daher ist eine Kombination beider Methoden anzustreben, da durch die quantitative Methode der zeitliche Verlauf des Überflutungsereignisses und durch die qualitative Methode die räumliche Variabilität berücksichtigt wird.

\section{Risikoanalyse}

An der urbanen Überflutungsvorsorge sind viele Akteure aus verschiedensten Fachdisziplinen beteiligt. Das Ziel ist 
die Identifizierung jener Stellen in einem Einzugsgebiet, welche das höchste Überflutungsrisiko aufweisen, um anschließend geeignete Gegenmaßnahmen zur Reduktion des Risikos ableiten zu können. Da nicht alle involvierten Personen über einen technischen Hintergrund verfügen, sollte die gewählte Methodik keine unnötige Komplexität aufweisen, um das Fehlerpotenzial aufgrund von Kommunikationsproblemen möglichst gering zu halten. Anhand der belastungsabhängigen Risikoanalyse wird das Überflutungsrisiko für ein vordefiniertes Schadensobjekt bestimmt. Dabei wird das Überflutungsrisiko in Risikoklassen eingeteilt, welche es erlauben, den komplexen Prozess der urbanen Überflutung einfach darzustellen. Dies ermöglicht eine einfache Kommunikation mit den jeweiligen Entscheidungsträgern sowie den betroffenen Bewohnern im Einzugsgebiet. Aus diesem Grund eignet sich eine Risikoanalyse sehr gut für die urbane Überflutungsvorsorge.

Aus wissenschaftlicher Sicht wird das Risiko durch eine Kombination aus der Gefahr für und der Vulnerabilität eines Objekts (Birkmann 2008; Scheid 2018) bestimmt. Dabei muss innerhalb der Risikodefinition die Auftrittswahrscheinlichkeit mitberücksichtigt werden. Im Fall der urbanen Überflutung wird diese durch die maximalen auftretenden Wiederkehrperioden eines Überflutungsereignisses, abgeleitet aus der Niederschlagswahrscheinlichkeit, berücksichtigt, die im Zuge der Simulationsszenarien definiert worden sind. Dabei ist für eine Berechnung des Überflutungsrisikos eine Überlagerung der Überflutungsgefahr und der Vulnerabilität des Schadensobjekts notwendig.

Als Schadensobjekt können Gebäude, Personen, Kulturgüter und die Umwelt definiert werden, wobei die negative Auswirkung auf Gebäude durch Überflutungen am häufigsten in der verfügbaren Literatur $\mathrm{zu}$ finden ist. In weiterer Folge wird das Schadenspotenzial des Schadensobjekts definiert und in Schadenspotenzialklassen eingeteilt. Die Gebäudenutzung ist für diese Einteilung die entscheidende Datengrundlage, um diese Klassifizierung durchführen zu können. Ferner wird die Gefahr der Überflutung anhand der Definition von Gefahrenklassen bewertet. Dabei werden die Modellergebnisse (Überflutungsflächen) des urbanen Überflutungsmodells als Basis für die Klassifizierung der Überflutungsgefähr- dung genutzt. Diese Klassifizierung des Schadenspotenzial sowie der Überflutungsgefährdung wird in Anlehnung an das DWA-Merkblatt 119 definiert (DWA 2016), wobei eine vierstufige Klassifizierung des Schadenspotenzials und der Überflutungsgefährdung empfohlen wird (Tab. 4).

An dieser Stelle muss festgehalten werden, dass die Klassifizierung der Überflutungsgefährdung auf Basis des erstellten Überflutungsmodells sehr genau durchgeführt werden kann, allerdings die Analyse des Schadenspotenzials höchstens eine qualitative und dabei subjektive Methode darstellt, welche stark von der regionalen Bebauungsstruktur abhängig ist (Unterkellerung, Heizsysteme, Bauausführung von Kellerfenstern und Eingangsbereichen). Daher sollte die Klassifizierung des Schadenspotenzials in jedem Einzugsgebiet kritisch von mehreren Fachdisziplinen beurteilt und im Anschluss unabhängig plausibilisiert werden.

Im Anschluss an die Klassifizierung des Schadenspotenzials sowie der Überflutungsgefahr kann das Überflutungsrisiko bestimmt werden. Dafür werden das Schadenspotenzial sowie die Überflutungsgefahr additiv überlagert. Durch die Einführung von Gewichtungsfaktoren können beide Eingangsgrößen in ihrer Ausprägung beeinflusst werden (Gl. 3, Scheid 2018). Dies ermöglicht eine variable Bestimmung des Überflutungsrisikos je nach vorliegender Situation. Es resultiert eine zweidimensionale Risikomatrix, die zur Risikobewertung des definierten Schadensobjekts (z.B. Gebäude) herangezogen wird. Das bestimmte Überflutungsrisiko muss anschließenden in Risikoklassen eingeteilt werden, wobei die Wiederkehrperiode des Überflutungsereignisses mitberücksichtigt werden muss. Dabei ist die Klassifizierung des Überflutungsrisikos mit aufsteigender Wiederkehrperiode in abgestufter Form durchzuführen (Abb. 6). Das Überflutungsrisiko wird bei einer hohen Eintrittswahrscheinlichkeit (im Beispiel $\mathrm{T}=10 \mathrm{a}$ ) höher bewertet als bei einer niedrigen Eintrittswahrscheinlichkeit (im Beispiel $\mathrm{T}=30 \mathrm{a}$ ), obwohl derselbe Wert für die Zustandsvariablen im Zuge der Risikoberechnung (Wasserstand und oder Fließgeschwindigkeit) berechnet wurde.

Diese Risikoklassen werden den Schadensobjekten (z.B. Gebäude) zugeordnet und in weiterer Folge mithilfe einer GIS-basierten Visualisierung die
Überflutungsrisikokarten erstellt. Diese bilden die Grundlage für die Bewertung und Diskussion der Überflutungen in einem urbanen Einzugsgebiet, sind die Basis für ein darauffolgendes Risikomanagement und daher der erste Schritt zu einer erfolgreichen urbanen Überflutungsvorsorge.

\section{Zusammenfassung}

Die Betrachtung von Flusshochwässern (fluvialen Überflutungen) ist innerhalb der Mitgliedstaaten der Europäischen Union durch die Hochwasserrisikomanagement-Richtlinie (Richtlinie 2007/60/EU) einheitlich geregelt, eine äquivalente Regelung für urbane Überflutungen (pluviale Überflutungen) existiert zu diesem Zeitpunkt noch nicht. Es obliegt derzeit den Kommunen selbst, wie sie mit der zunehmenden Problematik der urbanen Überflutungen umgehen. Eine einheitliche Regelung ist dabei jedenfalls anzustreben.

Der in diesem Beitrag vorgestellte Leitfaden zur modellbasierten urbanen Überflutungsvorsorge ist ein Werkzeug für Planer und Kommunen, um auf die Zunahme von urbanen Überflutungen reagieren zu können. Der Leitfaden wurde in insgesamt fünf Pilotstudien mit unterschiedlichsten räumlichen Strukturen angewandt. Die Erkenntnisse und Erfahrungen aus den jeweiligen Pilotstudien wurden in den finalen Leitfaden eingearbeitet, sodass eine praktische Umsetzbarkeit garantiert werden kann. Im Rahmen der Anwendung und Evaluierung durch Planungsbüros sowie Vertreter von Stadt- und Landesbehörden wurde die praktische Anwendbarkeit des Leitfadens bestätigt.

Der resultierende Leitfaden ist über den RAINMAN-Internetauftritt der Öffentlichkeit zugänglich (https://rain man-toolbox.eu).

Danksagung Die Erstellung des Leitfadens und die erwähnten Pilotstudien wurden durch das Amt der Steiermärkischen Landesregierung, Abteilung 14, Referat Schutzwasserbau beauftragt und finanziert, wobei das Projekt RAINMAN CE 986 mit Mitteln aus dem Interreg Central EuropeProgramm der Europäischen Union teilfinanziert wurde.

Funding Open access funding provided by Graz University of Technology. 
Open Access Dieser Artikel wird unter der Creative Commons Namensnennung 4.0 International Lizenz veröffentlicht, welche die Nutzung, Vervielfältigung, Bearbeitung, Verbreitung und Wiedergabe in jeglichem Medium und Format erlaubt, sofern Sie den/die ursprünglichen Autor(en) und die Quelle ordnungsgemäß nennen, einen Link zur Creative Commons Lizenz beifügen und angeben, ob Änderungen vorgenommen wurden.

Die in diesem Artikel enthaltenen Bilder und sonstiges Drittmaterial unterliegen ebenfalls der genannten Creative Commons Lizenz, sofern sich aus der Abbildungslegende nichts anderes ergibt. Sofern das betreffende Material nicht unter der genannten Creative Commons Lizenz steht und die betref- fende Handlung nicht nach gesetzlichen Vorschriften erlaubt ist, ist für die oben aufgeführten Weiterverwendungen des Materials die Einwilligung des jeweiligen Rechteinhabers einzuholen.

Weitere Details zur Lizenz entnehmen Sie bitte der Lizenzinformation auf http://creativecommons.org/licenses/ by/4.0/deed.de.
Bennett, N. D., Croke, B. F. W., Guariso, G., Guillaume, J. H. A., Hamilton, S. H., Jakeman, A. J. Marsili-Libelli, S., Newham, L. T. H., Norton, J. P., Perrin, C., Pierce, S. A., Robson, B., Seppelt, R., Voinov, A. A., Fath, B. D., and Andreassian, V. (2013): Characterising performance of environmental models. Environmental Modelling \& Software, 40, 1-20.

Berndtsson, R., Becker, P., Persson, A., Aspegren, H., Haghighatafshar, S., Jönsson, K., Larsson, R., Mobini, S., Mottaghi, M., Nilsson, J. Nordström, J., Pilesjö, P., Scholz, M., Sternudd, C., Sörensen, J., and Tussupova, K. (2019): Drivers of changing urban flood risk: A framework for action. Journal of Environmental Management, 240, 47-56.

Birkmann, J. (2008): Globaler Umweltwandel, Naturgefahren, Vulnerabilität und Katastrophenresilienz: Notwendigkeit der Perspektivenerweiterung in der Raumplanung. Raumforschung und Raumordnung, 66(1), 5-22. BMLFUW (2011): eHyd - Fachkarte Bemessungsniederschlag (Abteilung VII/3 - Wasserhaushalt, ed.), Vienna, Austria. Abgerufen 20.06.2020 [online] http://www.lebensministerium. at/wasser/wasser-oesterreich/wasserkreislauf/ hydrographische_daten/eHyd2008.html. BMLFUW (2016): Technische Richtlinie für die Gefahrenzonenplanungen gem. § 42a WRG, Wien.

Chimani, Heinrich, G., Hofstätter, M., Kerschbaumer, M., Kienberger, S., Leuprecht, A., Lexer, A. Peßsteiner, S., Poetsch, M. S., Salzmann, M. Spiekermann, R., Switanek, M., and Truhetz, H. (2016): Endbericht ÖKS15 - Klimaszenarien für Österreich - Daten - Methoden - Klimaanalyse, Wien. [online] https://data.ccca.ac.at/ dataset/endbericht-oks15-klimaszenarien-furosterreich-daten-methoden-klimaanalyse-v01 (Accessed February 4, 2021).

DWA (ed.) (2016): Risikomanagement in der kommunalen Überflutungsvorsorge für Entwässerungssysteme bei Starkregen, Hennef, Deutsche Vereinigung für Wasserwirtschaft, Abwasser und Abfall.

Europäisches Parlament (2007): Richtlinie 2007/60/EG des Europäischen Parlaments und des Rates vom 23. Oktober 2007 zur Schaffung eines Ordnungsrahmend für Maßnahmen der Gemeinschaft im Bereich der Wasserpolitik, Europäisches Parlament.

Fairfield, J. and Leymarie, P. (1991): Drainage networks from grid digital elevation models. Water Resources Research, 27(5), 709-717.

Field, C. B., Barros, V. R., and Intergovernmental Panel on Climate Change (eds.) (2014): Climate change 2014: impacts, adaptation, and vulnerability: Working Group II contribution to the fifth assessment report of the Intergovernmental Panel on Climate Change, New York, NY, Cambridge University Press.

GIS-Steiermark (2018): Gewässer und Wasserinformationen. Digitaler Atlas Steiermark. [on- line] https://gis.stmk.gv.at/atlas/(S(ulpyd4lmj0 xsn5jyma40vige) $/$ init. aspx? karte $=$ gew \&ks=das \& $\mathrm{cms}=$ da\&massstab $=800000$. Accessed December 8, 2018.

Guzman, J. A., Shirmohammadi, A., Sadeghi, A. M., Wang, X., Chu, M. L., Jha, M. K., Parajuli, P. B., Harmel, R. D., Khare, Y. P, and Hernandez, J. E. (2015): Uncertainty Considerations in Calibration and Validation of Hydrologic and Water Quality Models. Transactions of the ASABE 58(6), 1745-1762.

Hammond, M. J., Chen, A. S., Djordjević, S. Butler, D., and Mark, O. (2015): Urban flood impact assessment: A state-of-the-art review. Urban Water Journal, 12(1), 14-29.

Hawker, L., Bates, P., Neal, J., and Rougier, J. (2018): Perspectives on Digital Elevation Model (DEM) Simulation for Flood Modeling in the Absence of a High-Accuracy Open Access Global DEM. Frontiers in Earth Science, 6, 233.

Hunter, N. M., Bates, P. D., Neelz, S., Pender, G., Villanueva, I., Wright, N. G., Liang, D., Falconer, R. A., Lin, B., Waller, S., Crossley, A. J., and Mason, D. C. (2008): Benchmarking 2D hydraulic models for urban flooding. Proceedings of the Institution of Civil Engineers: Water Management, 161(1), 13-30.

Kim, Y., Eisenberg, D. A., Bondank, E. N., Chester, M. V., Mascaro, G., and Underwood, B. S. (2017): Fail-safe and safe-to-fail adaptation: decision-making for urban flooding under climate change. Climatic Change, 145(3-4), 397-412. Kirchengast, G., Kabas, T., Leuprecht, A., Bichler, $C_{\text {., }}$ and Truhetz, H. (2014): WegenerNet: A Pioneering High-Resolution Network for Monitoring Weather and Climate. Bulletin of the American Meteorological Society, 95(2), 227-242.

KLIWA (2019): Starkniederschläge Entwicklung in Vergangenheit und Zukunft - Kurzbericht, Arbeitskreis KLIWA

Maniak, U. (2016): Hydrologie und Wasserwirtschaft: eine Einführung für Ingenieure, Berlin Springer Vieweg.

Matzinger, A., Pilger, M. L., Nebauer, M., and Rouault, P. (2019): Potenzial von Bilddaten aus sozialen Medien für die urbane Überflutungsvorsorge - Versuch einer Anwendung für zwei extreme Starkregenereignisse in Berlin, in Aqua Urbanica, 6.

Mosley, M. P. and McKerchar, A. I. (1993): Chapter 8: Streamflow, in Handbook of Hydrology.

O'Donnell, E. C. and Thorne, C. R. (2020): Drivers of future urban flood risk. Philosophical Transactions of the Royal Society A: Mathematical, Physical and Engineering Sciences, 378(2168), 20190216.

ÖWAV (2009): ÖWAV-Regelblatt 11: Richtlinien für die abwassertechnische Berechnung und Dimensionierung von Abwasserkanälen.

Rauch, W., Bertrand-Krajewski, J.-L., Krebs, P.,

Mark, O., Schilling, W., Schütze, M., and Van-

rolleghem, P. A. (2002): Deterministic modelling of integrated urban drainage systems. Water Science and Technology, 45(3), 81-94.

Reinstaller, S., Maier, R., Jöbstl, C., Hornich, R. and Muschalla, D. (2020): Leitfaden - Modellbasierte Urbane Überflutungsvorsorge, Amt der Steirmärkischen Landesregierung A14 Wasserwirtschaft, Ressourcen und Nachhaltigkeit. [online] https://rainman-toolbox.eu/downloads/ (Accessed December 19, 2020).

Scheid, C. (2018): Gesamtkonzept einer GIS-basierten Starkregen-Risikoanalyse unter besonderer Berücksichtigung von Datenerfordernissen und methodischen erzielbaren Aussagefähigkeiten.

Schmitt, T. G., Thomas, M., and Ettrich, N. (2004): Analysis and modeling of flooding in urban drainage systems. Journal of Hydrology, 299(3-4), 300-311.

Smith, G. P., Davey, E. K., and Cox, R. (2014): Flood Hazard WRL Technical Report 2014/07, Australia, University of New South Wales. [online] https://www.aidr.org.au/media/2334/wrlflood-hazard-techinical-report-september2014.pdf. (Accessed December 14, 2020)

Statistik Austria (2020): Bevölkerung im Jahresdurchschnitt. Bevölkerung im Jahresdurchschnitt. [online] https://www.statistik.at/web de/statistiken/menschen_und_gesellschaft/be voelkerung/bevoelkerungsstand und veraende rung/bevoelkerung im jahresdurchschnitt/in dex.html. (Accessed December 07, 2020) UN (2018): $68 \%$ of the world population projected to live in urban areas by 2050 , says UN. [online] https://www.un.org/development/desa/ en/news/population/2018-revision-of-worldurbanization-prospects.html. (Accessed December 7,2020$)$

USDA (1986): Urban Hydrology for Small Watersheds - TR-55, Washington, DC, USA, United States Department of Agriculture - Natural Resources Conservation Service - Conservation Engineering Division.

Wang, Y., Chen, A. S., Fu, G., Djordjević, S. Zhang, C., and Savić, D. A. (2018): An integrated framework for high-resolution urban flood modelling considering multiple information sources and urban features. Environmental Modelling \& Software, 107, 85-95.

ZAMG (2020): Vermehrte Starkniederschläge? [online] https://www.zamg.ac.at $/ \mathrm{cms} / \mathrm{de} /$ klima/informationsportal-klimawandel/klima vergangenheit/neoklima/starkniederschlag (Accessed February 4, 2021).

Hinweis des Verlags Der Verlag bleibt in Hinblick auf geografische Zuordnungen und Gebietsbezeichnungen in veröffentlichten Karten und Institutsadressen neutral. 